JOURNAL OF

APPLIED

CRYSTALLOGRAPHY

ISSN 1600-5767

Received 24 December 2017

Accepted 3 March 2018

Edited by J. M. García-Ruiz, Instituto Andaluz de Ciencias de la Tierra, Granada, Spain

Keywords: optical mineralogy; threedimensional printing; birefringence; hands-on learning.

Supporting information: this article has supporting information at journals.iucr.org/j

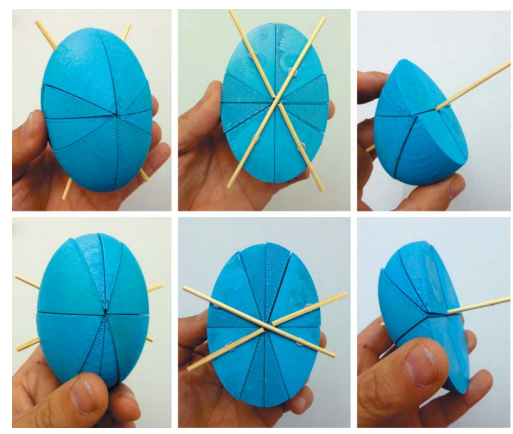

C 2018 International Union of Crystallography

\section{Three-dimensional-printing aids in visualizing the optical properties of crystals}

\author{
Lluís Casas* \\ Crystallography and Mineralogy Unit, Geology Department, Universitat Autònoma de Barcelona, Edifici C, Campus de la \\ UAB, Bellaterra, Barcelona 08193, Spain. *Correspondence e-mail: Iluis.casas@uab.cat
}

Crystal optics is an essential topic in mineralogy and is also relevant at postgraduate level in solid-state chemistry and physics. The emergence of lowcost three-dimensional-printing technologies makes possible the creation of tangible objects for multiple educational purposes. Within the field of crystallography and chemistry, some examples of applications of such educational objects have been recently published. These were intended for teaching and learning of crystal and molecular symmetry concepts. In this paper, three-dimensional-printing applications have been extended to crystal optics. A number of tangible models of optical indicatrices have been designed and printed. These models were conceived as dissection puzzles and allow students to actively work on assembling them and analyzing their geometrical features and relevant sections. The STL files of the presented models are made available with this paper.

\section{Introduction}

Petrological and mineralogical research is often carried out on polished materials and the polarizing light microscope is still the fundamental tool used for this purpose. Geology degrees generally include several practical courses that involve the use of the polarizing microscope, and this tool is also useful in many other areas like solid-state chemistry, materials science, archaeology, ceramology, art conservation and forensic science among others. Unfortunately, courses specifically devoted to the principles of crystal optics and to crystallography in general are on the decline in geology departments all over the world. This situation was pointed out 40 years ago (Donnay \& Donnay, 1978) and since then little has changed. Despite the decline, the polarizing microscope continues to be the most used tool for detailed study of rocks and minerals. The use of such microscopes will only give the best results if the user understands and applies the principles of crystal optics, but unfortunately only abridged sessions on such fields are regularly taught as part of basic mineralogy courses.

Crystal optics can be considered a branch of physics that originated from the description of the behavior of light when it propagates through anisotropic media, such as most minerals. Discoveries such as the solid-state laser have their origins in the principles of crystal optics (Tareen \& Kutty, 2001). To review the early contributions to optics the reader is referred to the introduction within the book by Laporte (2012). There are a number of good classical books for courses on crystal optics for mineralogists that have been repeatedly republished (e.g. Phillips, 1971; Gay, 1982; Bloss, 1999). These books emphasize optical theory and provide the fundamentals to understanding optical mineralogy, and their use should be 
complemented with training and colorful textbooks including exhaustive mineral descriptions and optical data (e.g. Barker, 2014). Unfortunately, in many geology colleges too often the theory is omitted to a great extent in favor of a practical prescriptive approach. The lack of theoretical concepts leaves the student unable to fully develop the skills and understanding of optical mineralogy. Basic contents of textbooks include the nature of light, isotropic and anisotropic media, and optical indicatrices; light-matter interaction (essentially simple and double refraction phenomena); and the polarizing microscope, its parts and adjustments (including the production of interference colors and interference figures). From these, optical indicatrices, interference colors and conoscopic microscopy (interference figures, optic sign, $2 V$ angles in biaxial media etc.) are considered the hardest parts by most students. A good comprehension of optical indicatrices is extremely useful for grasping all the other difficult parts and for a correct interpretation of phenomena observed with the polarizing microscope.

Advanced textbooks with mathematical developments to derive the indicatrices from the electromagnetic field equations and extensive use of tensors (Lipson et al., 2010) are used in highly specialized graduate courses on physics, physical optics or optical science. These are out of the scope of optical mineralogy and are hardly ever included as resources for crystal optics taught to undergraduate students of geology. Most of the recommended textbooks treat the indicatrices graphically including figures depicting axonometric projections of them and relevant sections to illustrate their use. Crystal optics is not really disappearing from undergraduate education on geology, but the condensed contents do not help the student to feel confident about the subject. They consider it difficult and many have unnecessarily complicated ideas about vibration directions, wavefronts, trajectories, interference colors and interference figures. One way to reinvigorate crystal optics in the classroom would be to replace or complement the static figures from the textbooks that can only be projected in the classroom by more dynamic components like interactive digital resources or physical models.

\section{Teaching optical indicatrices}

Students are commonly confused when first introduced to the phenomena of light propagation in anisotropic media. Noncubic crystalline materials exhibit a range of refractive indices instead of a discrete value. Every refractive index of a given material can be associated with a vibration direction of the light correlated with the corresponding velocity of the light vibrating in that direction. The optical indicatrix is a geometrical representation of the refractive indices of a crystalline solid in any vibration direction of light passing through it. The indicatrix is constructed in such a way that the refraction indices are plotted as radii that are parallel to the vibration direction of the light. Morphologically, the optical indicatrix of isotropic materials (cubic and amorphous solids) is spherical; that of high-symmetry (hexagonal, tetragonal and trigonal) anisotropic materials is a spheroid (or ellipsoid of revolution) with a single optical axis; and for low-symmetry (orthorhombic, monoclinic and triclinic) anisotropic materials the indicatrix is a triaxial ellipsoid bearing two optical axes.

The optical indicatrix is not merely used to visualize the range and variability of refractive indices of a given optical medium. The indicatrix, through the analysis of relevant sections of it, can help students to examine in detail the behavior of light passing through a solid medium. Many uses can be listed for this geometrical representation. The following are some of them: (i) it can be used to illustrate Neumann's principle (Nye, 1985) and to find the unbreakable link between the symmetry of any physical property of a crystal (exemplified by the refraction indices) and its structural symmetry; (ii) the optical axes of the indicatrices show how these optical paths do not produce birefringence and along them light behaves as in an isotropic material; (iii) the phenomenon of birefringence as well as the production of polarized light can also be understood using the optical indicatrix; (iv) birefringence produced by a given section of a material and its indicatrix is fundamental to comprehending the operation of polarizing microscopes, along with the concepts of optical sign, retardation, interference and interference color; (v) the concepts of ordinary and extraordinary light rays and their formation can also be understood using optical indicatrices; (vi) finally, birefringence can be analyzed in a very detailed way using indicatrices, as the vibration directions of the two light beams and their associated paths can be determined for any light beam entering the material in any direction.

Introductory courses on crystal optics usually include the notion of optical indicatrix from the very beginning. Students have to internalize the features of the two relevant types of indicatrices (uniaxial and biaxial) and their variants to fully exploit their use. This requires capacity for spatial visualization like in many other areas of crystallography. The use of physical models is well established for teaching other crystallographic concepts that require spatial visualization, such as point symmetry groups (Gremillion, 1982; Touret, 2004), or simply to aid with visualization of molecules and structures using common ball-and-stick models (Turner, 1971). However, for optical indicatrices the student usually has to rely on figures from specialized textbooks that commonly depict axonometric projections of the ellipsoids or sections of them along with their main relevant features in the case of solidlight interaction (Nesse \& Schultze, 2004) like optical axis, vibration directions, wave normal, wavefront and the direction of light rays. In the classroom hands-on experience on ellipsoids is usually restricted to soccer (isotropic ellipsoid) and rugby (uniaxial ellipsoid) balls; for the biaxial ellipsoid there is not an obvious analog (perhaps a compressed rugby ball) (Fig. 1). However, none of these objects can be sectioned for geometrical analyses.

Digital resources on optical indicatrices are rather scarce. A valuable interactive computational demonstration using Wolfram's computable document format was programmed by Danakas (2017). It allows the production of a three-dimensional virtual model of both the optic indicatrix and the 
normal surface (or $k$ surface) for any combination of principal refractive indices. This tool can be used to visualize and virtually manipulate an ellipsoid along with the corresponding optical axes, but it is intended to be used as a support material for physical optics rather than for more applied branches of optics like crystal or mineral optics. On YouTube it is possible to watch four short videos showing a three-dimensional animation of uniaxial and biaxial indicatrices of positive and negative optical sign (nightwach123, 2012) and other interesting videos that correlate indicatrix sections with the corresponding interference colors (Hirsch, 2017). These can be useful too, although they have no interactive capability. Finally it is worth mentioning that an online book on optical mineralogy by Gil Crespo (2016) includes very interesting interactive three-dimensional designs based on the figures that commonly appear in crystal optics textbooks.

Concerning tangible models of indicatrices, there are a number of pioneering projects using wood (Rogers, 1934) and sheet celluloid (Smith, 1938), and online make-yourself instructions are conveniently available too for updates of these models using acrylic sheets (Hirsch, 2003). All of these models consist of interlocked sections of the ellipsoids, and thus their surface is actually not present in the models. New low-cost three-dimensional-printing methods can improve and modernize these tangible models.

Additive manufacturing has become routinely available and three-dimensional-printing services can often be found in universities. Affordable printers for home users have also been introduced. Therefore, teaching resources that utilize three-dimensional printing is an emerging topic. Specifically, within the fields of crystallography and chemistry, there are recent papers on the production of tangible models of crystals (Casas \& Estop, 2015), unit cells (Moeck et al., 2014; Rodenbough et al., 2015), and atoms, molecules, bonds and orbitals (Griffith et al., 2016; Paukstelis, 2018; Smiar \& Mendez, 2016). The present paper enhances the list of three-dimensional printing teaching resources that pertain to crystal optics. A number of three-dimensional dissection puzzles of indicatrices are presented. These have already been used for teaching crystal optics to undergraduate students of geology from the
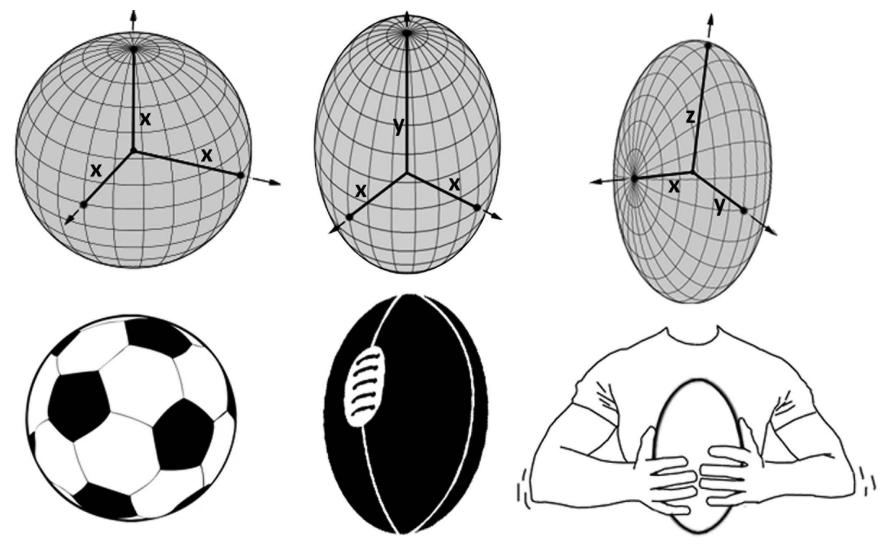

Figure 1

Representation of the isotropic (left), uniaxial (middle) and biaxial (right) indicatrices with the corresponding analogs (bottom).
Universitat Autònoma de Barcelona. The models can be scaled and printed in variable sizes. For classroom demonstrations large models are recommended, whereas for individual manipulation a smaller size (hand size) is more convenient. The corresponding printable STL files of the presented models are made available as supporting information.

\section{Models printed}

\subsection{Production process}

The printable STL files were obtained from designs made using the SolidWorks 2011 software (Onwubolu, 2011). Starting from a given ellipsoid, it is cut in the desired directions to produce the dissection puzzle. Taking into account that indicatrices are highly symmetric (for instance, they are all centrosymmetric), the dissection puzzles usually include some identical pieces and right-handed and left-handed versions of them.

Some holes were also designed in the middle of the planar faces of the pieces. The designed pieces were then printed using acrylonitrile butadiene styrene (ABS) filament on a UP Plus three-dimensional printer. Magnets were inserted within the holes on the planar faces (paying attention to their polarity) and pasted using an ABS glue. The magnets help the components of the assembled puzzle (i.e. the ellipsoid) to stay in place, but the desired section can be observed by detaching the puzzle into parts (Fig. 2).

Before assembly of the ellipsoids, the pieces were spray painted to disguise the magnets. Stickers and needles or toothpicks can be used to indicate the relevant directions (optical axis, vibration directions, wave normal and light paths).

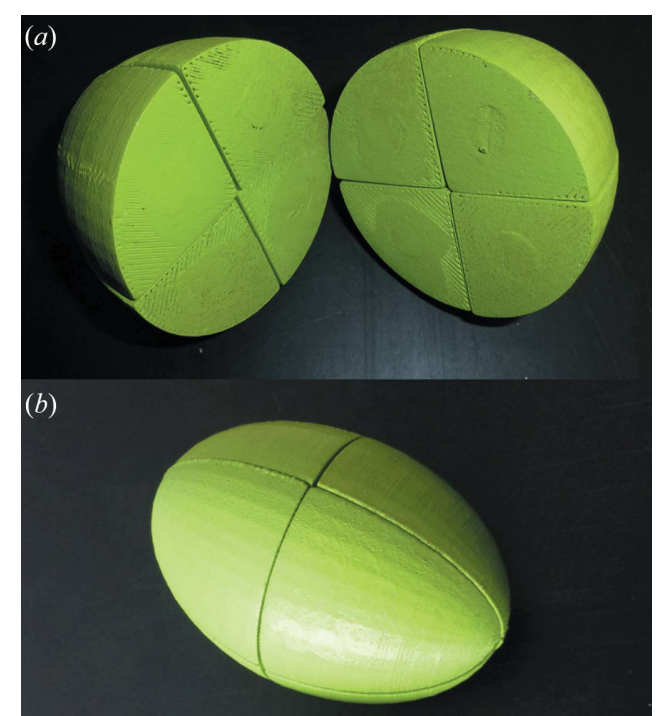

Figure 2

The indicatrices were produced as dissection puzzles that can be easily detached to observe a given section $(a)$ or fully assembled to get the ellipsoid shape $(b)$. 

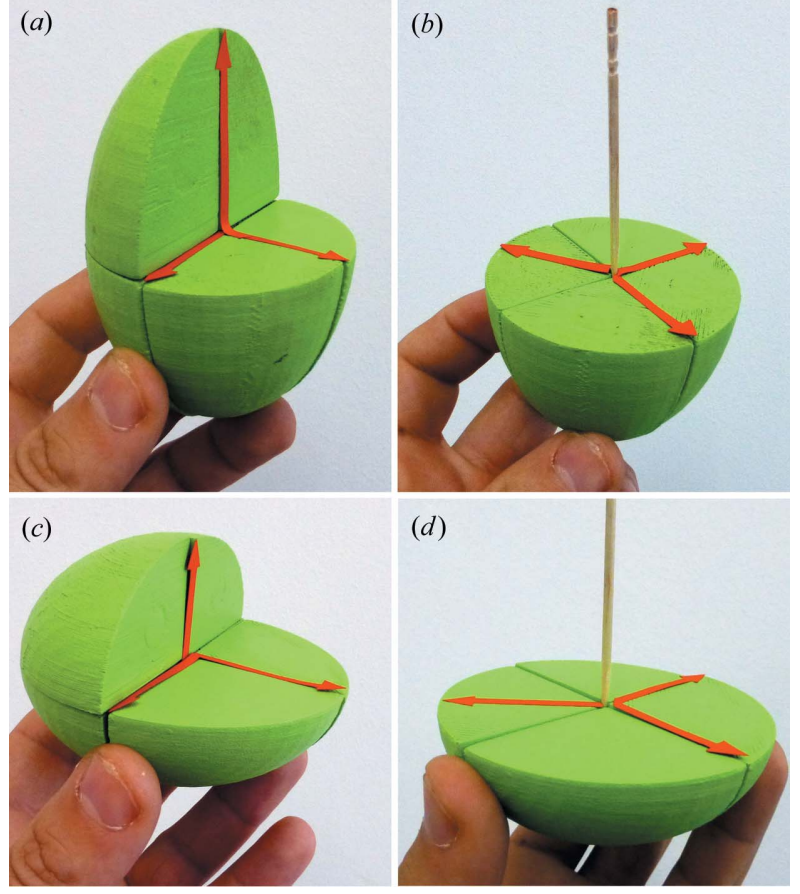

Figure 3

Printed uniaxial indicatrices showing the principal and the circular section for a $(a)$ prolate and $(c)$ oblate ellipsoid. The optical axis has been highlighted using a toothpick in $(b)$ and $(d)$.

\subsection{Uniaxial models}

Three types of uniaxial models have been designed and printed. They can be used not only to analyze the various types of sections in uniaxial ellipsoids but also to describe the interaction between light and optically uniaxial matter.

As one example, two dissection puzzles were made containing eight pieces that can be disassembled to show the single circular section and two orthogonal principal sections. These were produced in the shape of prolate (positive optical sign; Fig. 3a) and oblate (negative optical sign; Fig. 3c) ellipsoids. These models can be used to visualize the direction of isotropy and how a light ray entering the ellipsoid in the direction of the optical axis will not split into two different rays (Figs. $3 b$ and $3 d$ ). These models can also be used to illustrate the particular case of a ray with a normal wave perpendicular to the optical axis. In this case two ordinary rays are obtained. Both rays share the same normal wave and ray direction, but they vibrate in perpendicular directions (one of them in the direction of the optical axis) with different associated refraction indices and therefore with different velocities. In the case of an oblate ellipsoid the ray that vibrates along the optical axis direction is the fastest, in contrast to a prolate ellipsoid which has the slowest ray in that direction (Fig. 4).

A different dissection puzzle of a prolate ellipsoid was made containing 12 pieces. This can be disassembled to show the circular section and one principal section and two additional arbitrary (but mutually orthogonal) elliptical sections (Fig. $5 a$ ). This model could also be used to visualize the direction of isotropy but it is especially designed to illustrate the general case of a random wave normal. The wave normal coincides with the trajectory of a light ray entering the anisotropic material from an isotropic medium (like air, glass or resin). For an incident angle of $0^{\circ}$ (i.e. a light ray entering perpendicular to the surface of the material, as it is in the usual transmission geometry of a polarizing microscope), this direction is also the wave normal of the two rays produced by birefringence. A section perpendicular to the wave normal can be shown to locate the two vibration directions corresponding to the two refracted rays as they are parallel to the axes of the elliptical section (Fig. 5b). Finally, the directions of the two rays can also easily be identified by disassembling the ellipsoid in the right sections containing one of the two vibration directions and the shared wave normal. For one of these sections the vibration direction is an axis of the elliptical section. The corresponding conjugate direction is therefore perpendicular to it and coincides with the wave normal (Fig. 5c). In this case, as wave normal and ray direction coincide, the ray is ordinary (this ray behaves as though it were in an isotropic material). In contrast, for the other section the vibration direction is not an axis of the elliptical section, and therefore the conjugate direction (i.e. the ray direction) does
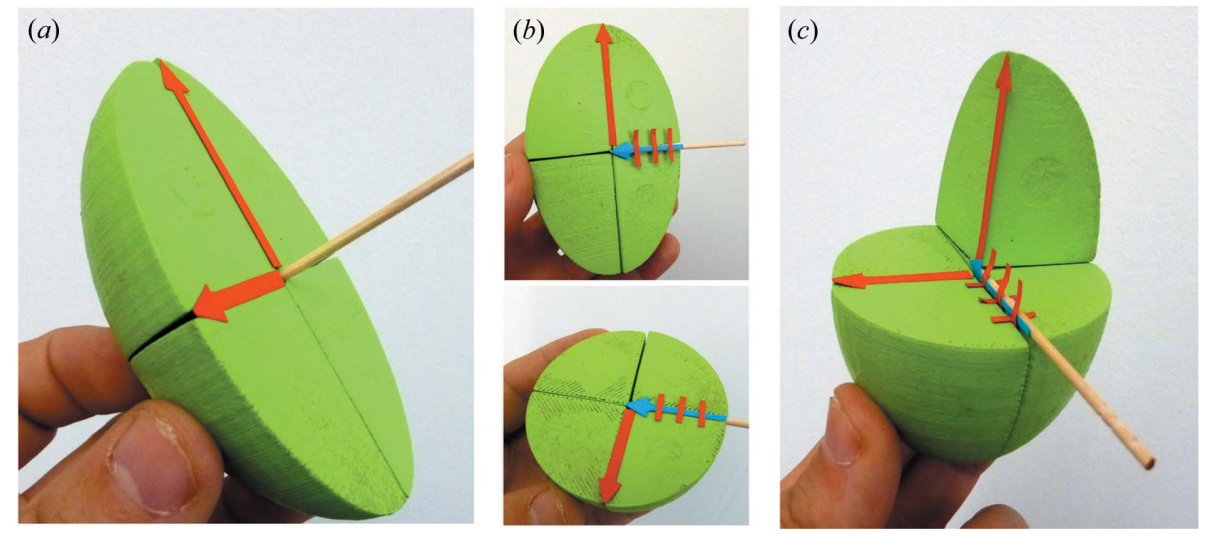

Figure 4

Splitting of a light ray entering perpendicularly to the optical axis of a uniaxial medium. (a) The toothpick is the wave normal of the incident ray (and also that of the two refracted rays); the red arrows indicate the vibration directions of the refracted rays. (b) The blue arrows indicate the directions of the refracted rays (they coincide). (c) Another view of the model, where it is apparent that both rays are ordinary and they share the same trajectory though they travel at different speeds. 

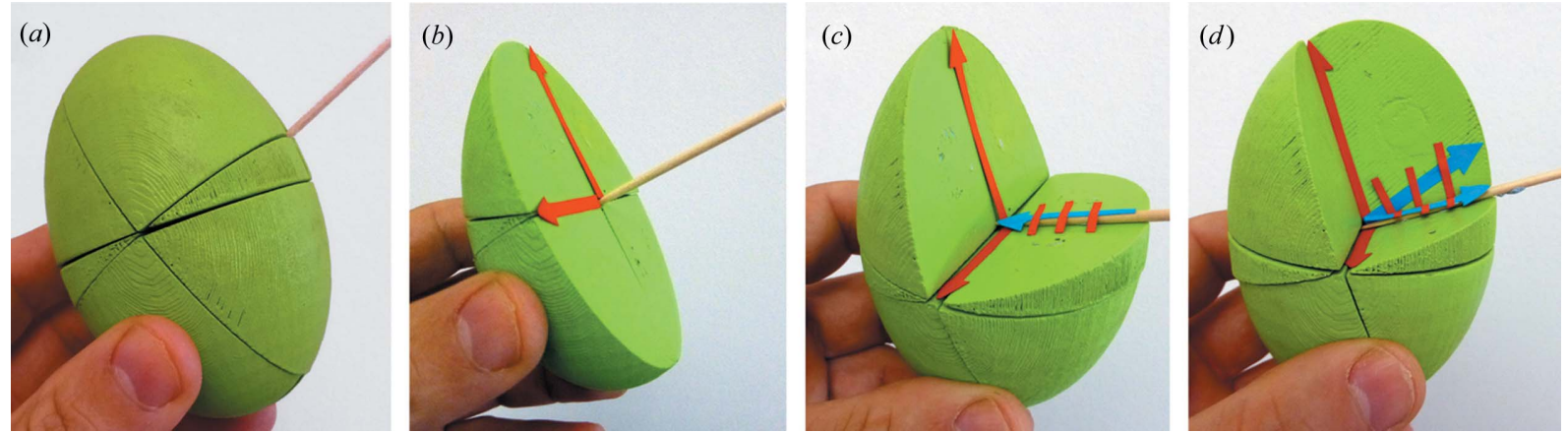

Figure 5

General case of splitting of a light ray entering a uniaxial medium. The red arrows indicate the vibration directions and the blue arrows the light directions. One of the rays is ordinary [see $(c)$ ], and the other one is extraordinary.

not coincide with the wave normal and the ray is called extraordinary (Fig. 6). The refraction indices 'associated' with the two rays are indicated by the length of the vibration directions within the ellipsoid. For this 12-piece model (prolate ellipsoid) the extraordinary ray has a higher index than the ordinary one and therefore it would correspond to an optically positive uniaxial material (Fig. $5 d$ ).
Finally, an additional dissection puzzle of a prolate ellipsoid was also made. This is a similar model to that shown in Fig. 4, but in this design the model can be placed inside a hexagonal prism that can also be sectioned like the ellipsoid (Fig. 7). This can be used to exemplify the relationship between the crystal morphology and the optical ellipsoid, i.e. Neumann's principle.


Figure 6

Graphical procedure to obtain the direction of the extraordinary ray in a uniaxial medium. A halfellipsoid $(a)$ can be used to draw on a sheet of paper $(b)$ the ellipse, where the vibration direction can be used to calculate the direction of the ray (i.e. the conjugate direction). A blue arrow (c) has been stuck on the section of the model and small stickers indicate the vibration direction of this extraordinary ray.
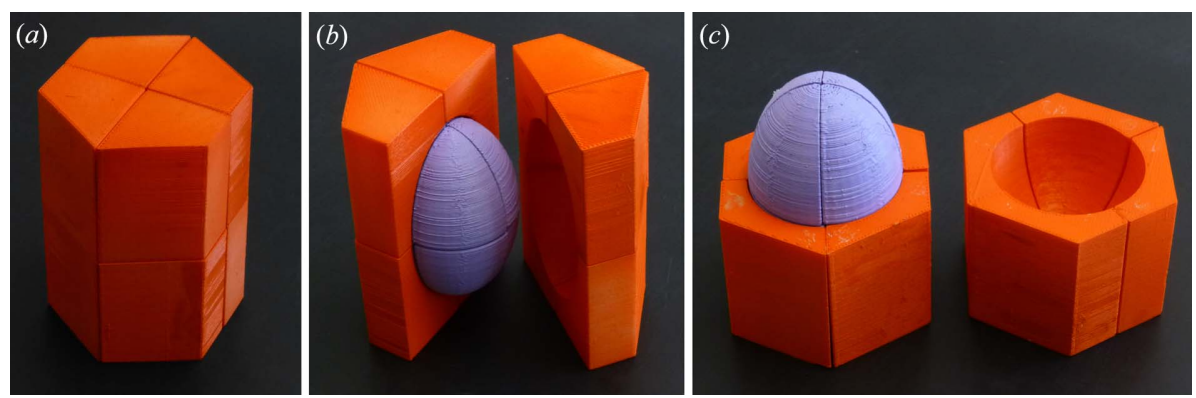

Figure 7

Uniaxial ellipsoid inside a hexagonal prism. The optical axis (revolution axis) has to coincide with the hexagonal axis of the prism.

\subsection{Biaxial models}

Five types of biaxial models have been designed and printed.

A triaxial ellipsoid was designed as an eight-piece dissection puzzle. For this design there are only two shapes of pieces and they are enantiomorphic versions of the same morphology. One of the uses of this model is to show the three principal sections of a biaxial ellipsoid (the $X Y, X Z$ and $Y Z$ planes) and to highlight the $\mathrm{mmm}$ symmetry of these types of ellipsoids (Fig. 8). The model can also be used to illustrate the case of a ray entering a biaxial optical medium along the direction of one of the ellipsoid axes. The observation of the relevant sections can help to illustrate that the vibration directions of the two rays produced by birefringence will have the refraction indices 'associated' with the other two axes of the triaxial indicatrix and that again, as in a previously seen case, two ordinary rays are obtained. (Fig. 9).

Two more ellipsoids were produced consisting of 16 pieces (Figs. $10 a$ and $10 d)$. These can be disassembled to show the $X Z$ principal section and the two inclined intersecting circular sections. The directions perpendicular to the two circular sections are the optical axes and the $X Y$ section can be 

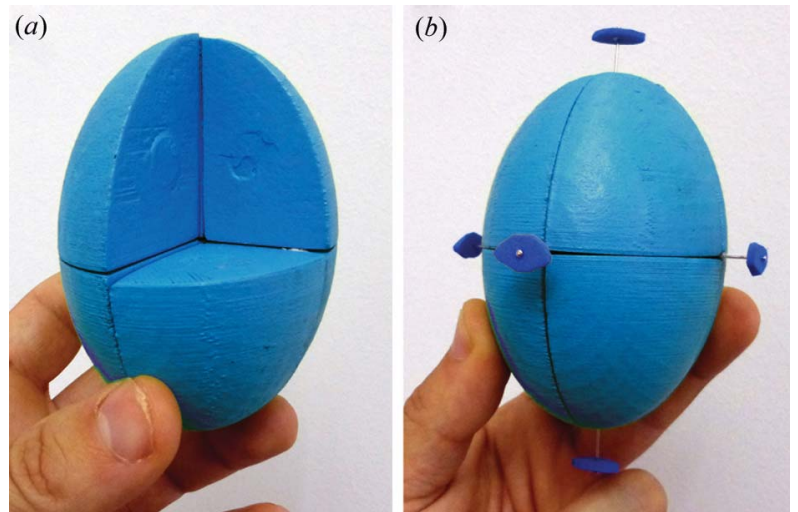

Figure 8

Biaxial model sectioned to illustrate its mirror planes; in $(b)$ the twofold rotation axes have been highlighted.

used to visualize the angle between the two optic axes (i.e. the optic angle or $2 V$ angle). One of the 16-piece ellipsoids was designed having $Z$ as the acute bisectrix of the $2 V$ angle (i.e. an optically positive biaxial indicatrix; Fig. $10 a$ ) and the other one as having $X$ as the acute bisectrix of the $2 V$ angle (i.e. an optically negative biaxial indicatrix; Fig. 10d). Therefore these ellipsoids can be used to show the two circular sections of any triaxial ellipsoid and the corresponding optical axes and their associated direction of isotropy (Figs. $10 c$ and 10f). Additionally, the concepts of $2 \mathrm{~V}$ angle and the definition of optical sign in biaxial media can be illustrated (Figs. $10 b$ and 10e).

Another eight-piece dissection puzzle was printed, which can be disassembled to show the $X Z$ and $Y Z$ principal sections and an additional section cut perpendicular to the $X Z$ plane and at a random angle with the $Y Z$ plane (Fig. 11a). This can be used to illustrate how, at certain incident angles, birefringence can also result in a pair of extraordinary and ordinary rays in biaxial media. Any ray with a wave normal parallel to one of the three principal sections (but not collinear with any axis) will split into two rays that share the same wave normal (considering an incident angle of $0^{\circ}$ ). One of the obtained rays is ordinary and vibrates along the axis not contained in the considered principal section (Fig. 11b); the other ray is extraordinary and vibrates on the principal section and at a certain angle with respect to the shared wave normal. Using the model we can consider a wave normal contained in the $X Z$ plane at an angle of about $15^{\circ}$ with respect to the $X$ axis. Then the ordinary ray vibrates along the $Y$-axis direction and travels along the wave normal. According to

Figure 10
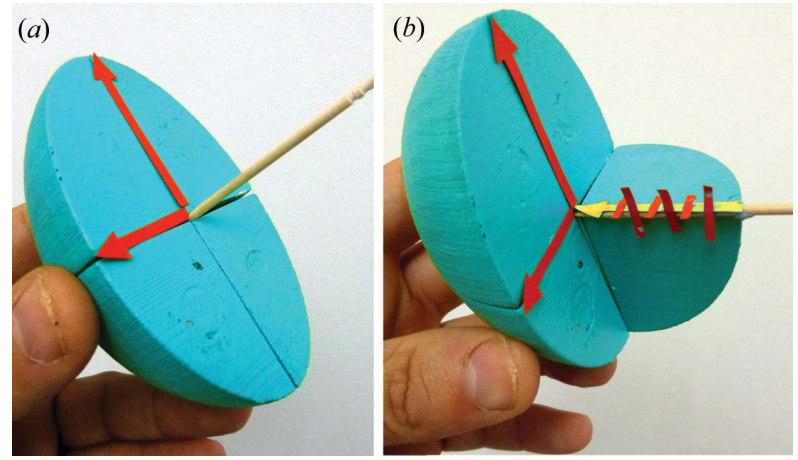

Figure 9

Biaxial model exhibiting the case of a ray entering along one of the ellipsoid axes, producing two ordinary rays.

the shape of the model this is the fastest ray. The slowest in this case is the extraordinary ray that vibrates on the $X Z$ plane (perpendicular to the wave normal) and travels also along this plane at an angle of about $20^{\circ}$ to the wave normal.

Finally, another eight-piece dissection puzzle was produced, exhibiting three mutually perpendicular sections randomly oriented (i.e. none of them is a principal or a circular section although one of the elliptical sections is cut along its two semiaxes; Fig. 12a). This model can be used to illustrate the general case of a light ray entering the biaxial medium in a random direction. Here, again, any light ray will split into two rays that share the same wave normal (considering an incident angle of
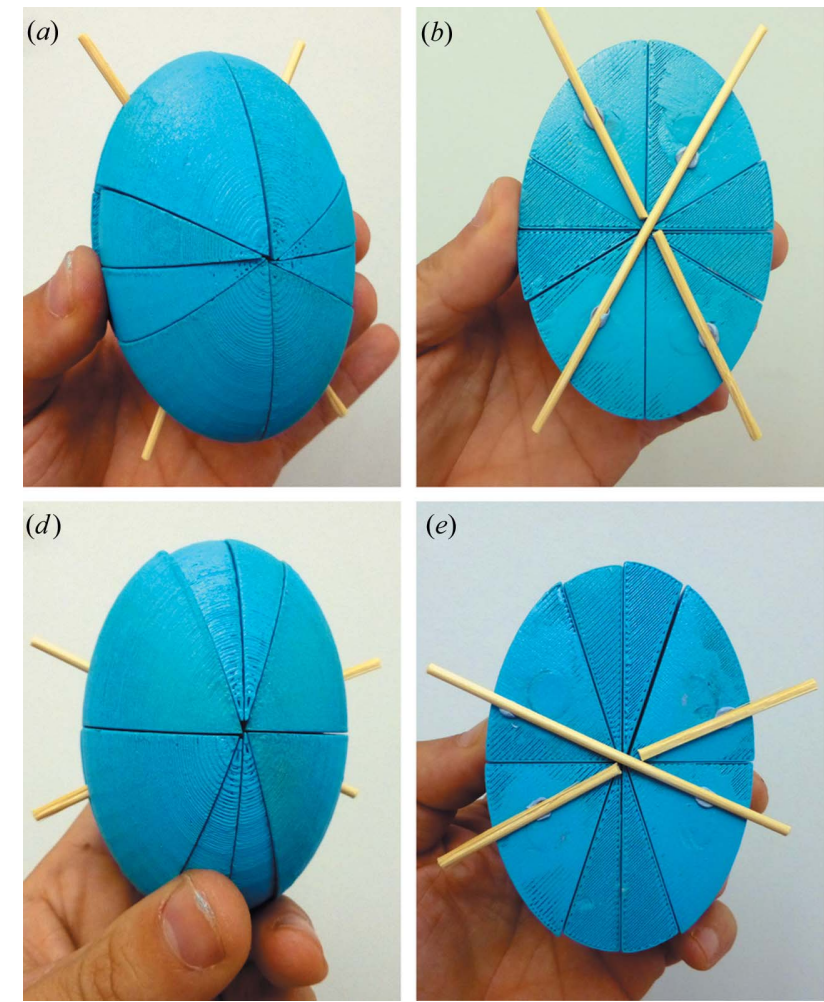

(e)

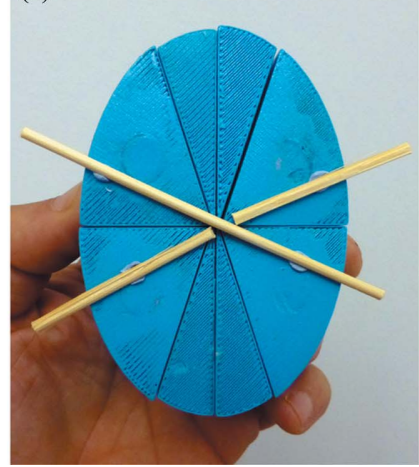

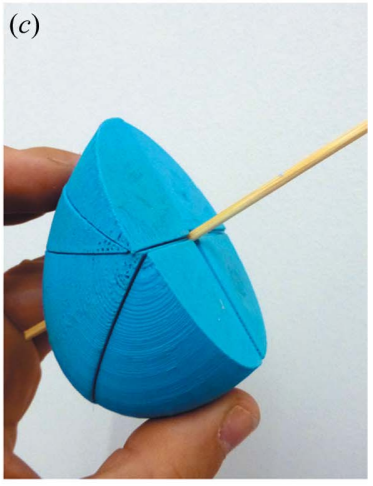

$(f)$

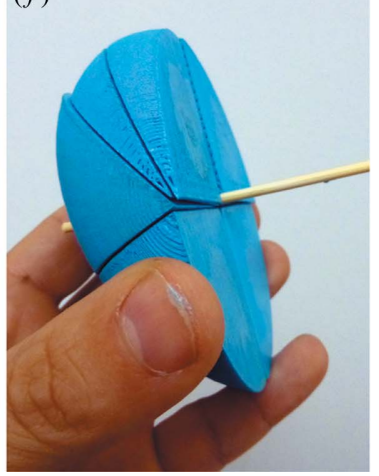

Biaxial models, optically positive (top) and negative (bottom). The optical axes have been highlighted using wooden sticks. Besides the principal sections, the models can be disassembled along the circular sections. 

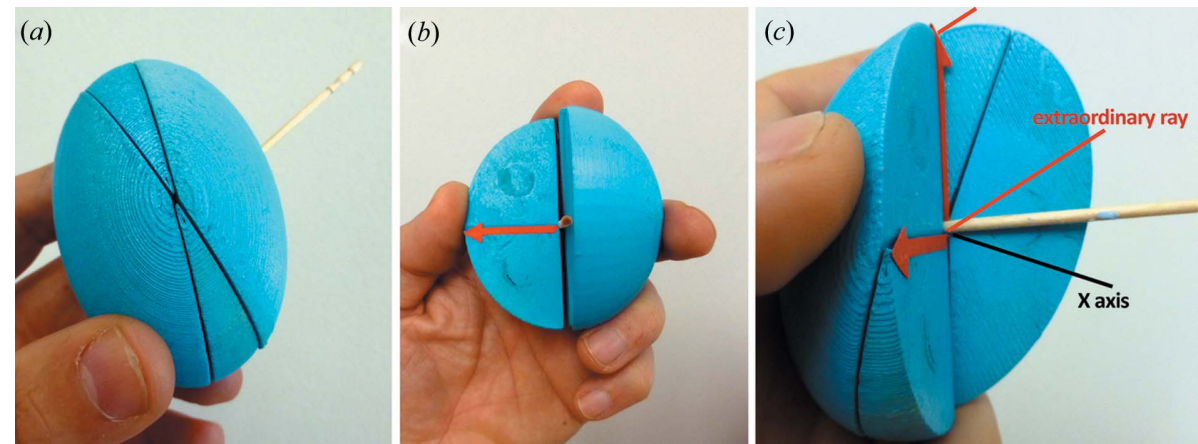

Figure 11

Biaxial model. A toothpick has been used to indicate a wave normal parallel to one of the three principal sections (but not collinear with any axis). One of the refracted rays is ordinary $(b)$ and its path coincides with the wave normal. The other one is extraordinary $(c)$ and its direction has to be calculated as a conjugate direction.
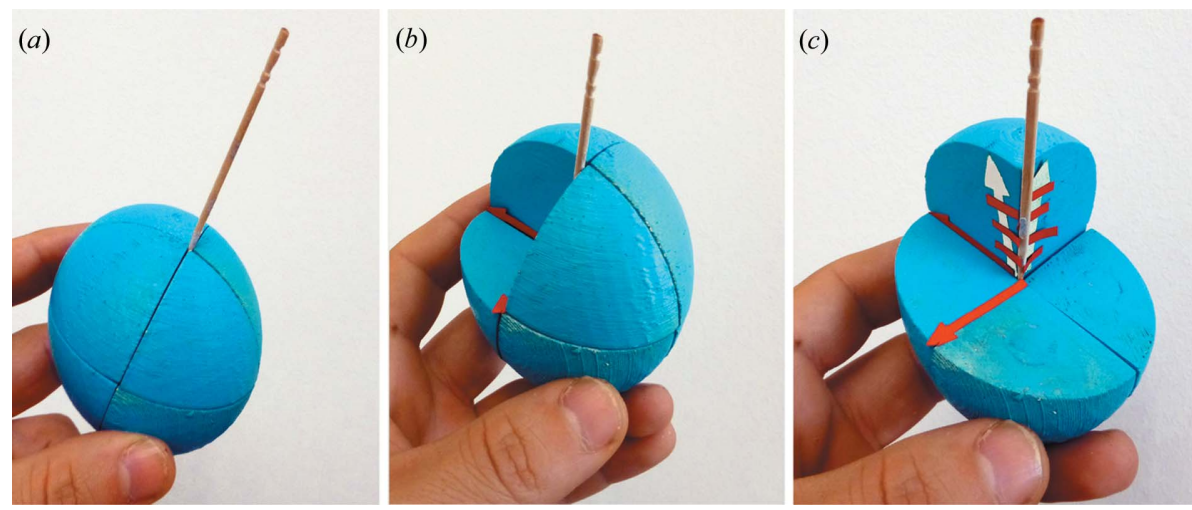

Figure 12

Biaxial model that can be used to illustrate the general case of birefringence in a biaxial medium. The wavefront is an ellipse whose semiaxes indicate the vibration directions of the refracted rays [red arrows in $(b)$ and $(c)$ ]. The directions of both rays can be calculated in the corresponding elliptical section as conjugate radii [white arrows in $(c)$ ]. Both rays are extraordinary.

$0^{\circ}$; Fig. $\left.12 b\right)$. Considering the two sections containing the wave normal and each of the vibration directions it is apparent that neither of the two vibration directions, or the wave normal, is oriented along the semiaxes of the elliptical section of the indicatrix containing both the wave normal and the corresponding vibration directions. Then the trajectories of the two rays have to be calculated as the conjugate direction of the vibration directions on the corresponding elliptical sections (Fig. 12c). Therefore both rays are extraordinary.

\section{Conclusion}

Different three-dimensional dissection puzzles of optical indicatrices have been presented. These can be used as support materials to study a number of crystal optics concepts (optical indicatrix, Neumann's principle, birefringence, optical isotropy directions, optical axes, optical sign, retardation, ordinary and extraordinary light rays, etc.). The presented puzzles are only examples of the vast potential of threedimensional design and printing to afford students and teachers with additional resources to overcome the difficulties of learning and teaching concepts in optics that require capacity for abstraction and spatial visualization.
Nine types of indicatrices as described above (four uniaxial models and five biaxial models) were designed as dissection puzzles. These are made freely available as printable STL files that can be found in the supporting information. Educators are encouraged to use and adapt these models in their lectures.

\section{Acknowledgements}

Martí Vinyes is acknowledged for giving the idea to produce indicatrices using three-dimensional printers. Anna Anglisano is also recognized for suggesting the use of magnets to attach the puzzle pieces. Finally, Àngels Canals is acknowledged for pointing out the interest of combining crystal morphology and an optical ellipsoid in a single design.

\section{References}

Barker, A. J. (2014). A Key for Identification of Rock-Forming Minerals in Thin Section. Boca Raton: CRC Press.

Bloss, F. D. (1999). Optical Crystallography. Washington, DC: Mineralogical Society of America.

Casas, L. \& Estop, E. (2015). J. Chem. Educ. 92, 1338-1343.

Danakas, S. (2017). Uniaxial-Biaxial Birefringence: Geometrical Constructions for Optical EM Waves, http://demonstrations.wolfram. com/UniaxialBiaxialBirefringenceGeometricalConstructionsForOptic/. 
Donnay, G. \& Donnay, J. D. H. (1978). Am. Mineral. 63, 840-846.

Gay, P. (1982). An Introduction to Crystal Optics. London: Longman.

Gil Crespo, P. P. (2016). Atlas de Mineralogía Óptica, http://www. ehu.eus/mineralogiaoptica/Atlas_de_Mineralogia_Optica/Atlas_ de_Mineralogia_Optica.html.

Gremillion, A. F. (1982). J. Chem. Educ. 59, 194.

Griffith, K. M., de Cataldo, R. \& Fogarty, K. H. (2016). J. Chem. Educ. 93, 1586-1590.

Hirsch, D. (2003). New Indicatrices, https://davehirsch.com/other/ indicatrices/index.html.

Hirsch, D. (2017). Uploaded Videos on Indicatrices, https://www. youtube.com/channel/UCkJBdQsfAfzLKtL6rHxkHwA.

Laporte, O. (2012). Optics. Amsterdam: Elsevier.

Lipson, A., Lipson, S. G. \& Lipson, H. (2010). Optical Physics. Cambridge University Press.

Moeck, P., Stone-Sundberg, J., Snyder, T. J. \& Kaminsky, W. (2014). J. Mater. Educ. 36, 77-96.

Nesse, W. E. \& Schultze, D. J. (2004). Introduction to Optical Mineralogy. Oxford University Press. nightwach123 (2012). Uploaded Videos on Indicatrices, https://www. youtube.com/user/nightwach123.

Nye, J. F. (1985). Physical Properties of Crystals: Their Representation by Tensors and Matrices. Oxford: Clarendon Press.

Onwubolu, G. (2011). A Comprehensive Introduction to SolidWorks 2011. Mission: SDC Publications.

Paukstelis, P. J. (2018). J. Chem. Educ. 95, 169-172.

Phillips, W. R. (1971). Mineral Optics: Principles and Techniques. San Francisco: W. H. Freeman \& Co.

Rodenbough, P. P., Vanti, W. B. \& Chan, S.-W. (2015). J. Chem. Educ. 92, 1960-1962.

Rogers, A. F. (1934). Am. Mineral. 19, 206-208.

Smiar, K. \& Mendez, J. D. (2016). J. Chem. Educ. 93, 1591-1594.

Smith, H. T. U. (1938). Am. Mineral. 23, 629-643.

Tareen, J. A. K. \& Kutty, T. R. N. (2001). A Basic Course in Crystallography. Hyderabad: Universities Press.

Touret, L. (2004). Dutch Pioneers of the Earth Sciences, edited by K. N. A. van Wetenschappen, pp. 43-58. Amsterdam: Royal Netherlands Academy of Arts and Sciences.

Turner, M. (1971). J. Chem. Educ. 48, 407. 